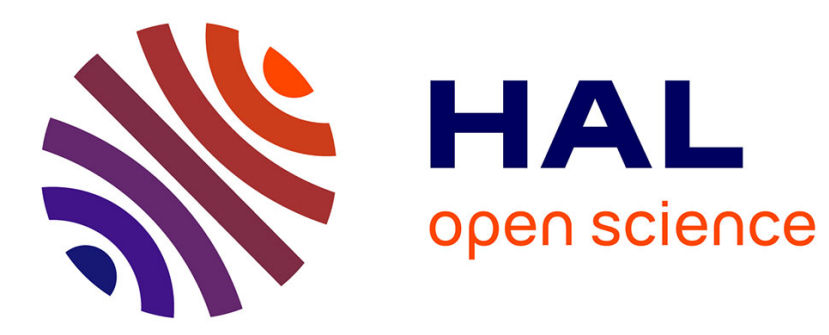

\title{
Halal carcéral
}

Claire de Galembert, Corinne Rostaing, Céline Béraud

\section{To cite this version:}

Claire de Galembert, Corinne Rostaing, Céline Béraud. Halal carcéral. Les sens du halal: une norme dans un marché mondial, CNRS éditions, pp.317-340, 2015, 978-2-271-08156-8. 10.4000/books.editionscnrs.24633 . halshs-01297649

\section{HAL Id: halshs-01297649 https://shs.hal.science/halshs-01297649}

Submitted on 4 Apr 2016

HAL is a multi-disciplinary open access archive for the deposit and dissemination of scientific research documents, whether they are published or not. The documents may come from teaching and research institutions in France or abroad, or from public or private research centers.
L'archive ouverte pluridisciplinaire HAL, est destinée au dépôt et à la diffusion de documents scientifiques de niveau recherche, publiés ou non, émanant des établissements d'enseignement et de recherche français ou étrangers, des laboratoires publics ou privés. 


\section{Halal carcéral}

Claire de Galembert*, Corinne Rostaing*, Céline Béraud*

Comment s'exprime la demande de prise en compte de la normativité islamique de la part des détenus en matière alimentaire ? Quelles sont les réponses apportées par les différents acteurs concernés, aussi bien du côté des détenus que de l'institution et ses personnels ? La présente contribution se propose d'apporter quelques éléments de réponse à ces questions en prenant appui sur une recherche relative au fait religieux en prison récemment achevée. Nous nous cantonnerons cependant ici à la seule dimension alimentaire du halal1. Cette recherche2 (Voir encadré) n'a - et c'est important de le préciser - pas eu pour objet le halal en tant que tel. Elle ne s'est pas davantage concentrée sur l'islam puisqu'elle a concerné l'ensemble des religions.

${ }^{*}$ Claire de GALEMBERT (ISP, Paris), Chargée de recherche CNRS à l'Institut des Sciences Sociales du Politique (ISP)- ENS de Cachan, Galembert@ens-Cachan.fr.

* Corinne ROSTAING (Centre Max Weber, Lyon), Sociologue, Maitre de conférences HDR à l'université Lyon 2, Corinne.Rostaing@univ-lyon2.fr.

* Céline BERAUD (CERReV), Sociologue, Maître de conférences à l'université de Caen, Celine.Beraud@gmail.com.1. Etant entendu que le Halal dépasse aujourd'hui que la question de l'alimentation conforme aux prescriptions de l'abattage rituel (Bergeaud-Blacker, 2012).

2. Céline Béraud, Claire de Galembert et Corinne Rostaing, Des Dieux et des hommes en prison. Rapport remis à la Direction de l'Administration Pénitentiaire, Mai 2013. 
Comment s'exprime et se configure la normativité islamique en prison et quelles sont les réponses apportées à cette exigence en matière alimentaire par les différents acteurs concernés, qu'il s'agisse des détenus ou de l'institution et de ses personnels ? Cette contribution propose quelques éléments de réponse à ces questions en se centrant donc exclusivement sur la question alimentaire, étant entendu que le halal excède aujourd'hui largement cette question ${ }^{1}$. Elle s'appuie sur une recherche sur le fait religieux en prison récemment achevée. Cette recherche ${ }^{2}$ (voir encadré) n'a et c'est important de le préciser - pas eu pour objet le halal en tant que tel. Elle ne s'est pas davantage concentrée sur l'islam puisqu'elle a concerné l'ensemble des religions.

Commanditée par la Direction de l'Administration pénitentiaire (DAP), cette recherche ethnographique, menée dans huit prisons (aussi bien des établissements pour peine que des maisons d'arrêt), nous a conduit à mener des observations (notamment dans les cuisines ou la préparation des cantines) et à réaliser des entretiens avec 15 aumôniers musulmans (aumôniers locaux, régionaux et l'aumônier national), 70 détenus de confession musulmane et 22 surveillants se réclamant de l'islam. Cet échantillon n'a aucune ambition de représentativité. Faute de statistiques religieuses (Dargent, 2010), il est d'ailleurs difficile de quantifier la part de l'islam, pourtant réputé «première religion carcérale». À titre indicatif en 2012, l'administration pénitentiaire a recensé 12000 détenus déclarant vouloir faire le Ramadan (sur 68000 détenus environ)³.

Notre échantillon comprend toutefois un nombre non négligeable d'interviewés se revendiquant de l'islam (74), cette islamité allant, selon les personnes, de la stricte observance à un rapport plus distancé, assimilant l'islam à une culture plus qu'à une religion. Encore faut-il préciser que nos guides d'entretiens élaborés sur la base du plus petit dénominateur commun entre les diverses religions malgré des questions relatives aux contraintes religieuses en termes d'alimentation ne spécifiaient pas le halal. Le sujet n'a toutefois pas manqué d'apparaitre sans sollicitation directe

\footnotetext{
${ }^{1}$ Etant entendu que le Halal dépasse aujourd'hui que la question de l'alimentation conforme aux prescriptions de l'abattage rituel (Bergeaud-Blacker, 2012).

${ }^{2}$ Céline Béraud, Claire de Galembert et Corinne Rostaing, Des Dieux et des hommes en prison. Rapport remis à la Direction de l'Administration Pénitentiaire, Mai 2013.

${ }^{3}$ Le nombre de détenus musulmans reste approximatif sachant que nombre de détenus font le ramadan sans s'inscrire dans ce dispositif tandis que d'autres s'inscrivent pour des raisons qui n'ont souvent rien avoir avec la religion. On est donc loin des 50 ou $70 \%$ de musulmans évoqués régulièrement par la presse.
} 
et l'on peut considérer méthodologiquement nos observations d'autant plus significatives que la question a émergé spontanément.

Le premier enseignement de cette enquête réside dans le constat que le halal - au sens de prescriptions religieuses relatives à l'alimentation-n'est pas un objet majeur de revendication de la part des détenus musulmans. Présente cette revendication ne s'exprime du moins pas de manière aussi centrale que les demandes relatives au culte collectif ou aux visites des aumôniers en cellule, encore très aléatoires dans certains établissements. Même lorsque la question de la discrimination est mentionnée, le halal reste peu évoqué se cantonnant à quelques remarques ponctuelles dénonçant un traitement différentiel des juifs et des musulmans. De manière significative le sujet apparaît également peu dans nos entretiens avec les aumôniers musulmans : deux sur huit seulement l'ont évoqué. Le sujet apparaît enfin rarement dans les entretiens avec les personnels si l'on met à part ceux qui sont en charge des cuisines et des cantines.

Cette place relative du halal dans nos entretiens peut tenir à l'effet inhibiteur de l'incarcération sur l'expression de la normativité islamique, cet effet jouant à plusieurs niveaux. En premier lieu, le contexte, assimilé à l'impur, peut rendre la problématique halal/haram moins pertinente ou légitime : des détenus de tradition islamique ont souligné d'ailleurs le caractère paradoxal de l'investissement de certains de leur coreligionnaires dans une stricte observance au regard de leur statut de délinquant ou de criminel. En second lieu, les contraintes liées à l'incarcération sont considérées par certains comme un motif religieusement valable de suspension de l'exigence de respect des normes religieuses en matière d'alimentation. Enfin, le caractère encore très limité de la prise en charge de l'islam par l'institution relègue cette question au rang de question secondaire en comparaison d'autres considérations religieuses plus prioritaires évoquées ci-dessus. Ce à quoi peut venir s'ajouter une logique d'autocensure de la part de certains détenus musulmans qui hésitent à se montrer «trop musulmans » de peur d'être considérés comme « radicaux ».

Mais ces effets inhibiteurs ne doivent pas être surestimés. L'enfermement n'empêche pas le développement de pratiques allant dans le sens de l'islamisation de l'alimentation (Bergeaud- 
Blacker, 2012). «Ombre portée » du monde (Fassin 2015), la prison n'est pas un monde hors de la société. Et quelles que soient les contraintes de l'incarcération, on y observe comme ailleurs l'apparition d'un islamic way of life s'exprimant aussi bien à travers les mises en scène de soi (par le vêtement, le marquage du corps ou la gestuelle) que des conduites marquant une différenciation au nom de la religion. Si la prison freine l'emprise des normes islamiques sur certains détenus, elle ne l'empêche pas. Elle peut même chez certains l'exacerber, l'investissement dans la religion constituant pour une partie minoritaire de détenus une ressource pour surmonter l'épreuve carcérale.

Le monde pénitentiaire n'échappe donc pas, comme d'autres administrations (Papi, 2012), aux effets de la transformation des modes de consommation des personnes se référant à l'islam (Bergeaud-Blackler, Bernard, 2010 ; Kepel, 2011). La DAP, qui s'acquitte a minima de ses obligations en matière de respect de la liberté religieuse (en comparaison avec certains États européens voisins), se trouve aujourd'hui confrontée à une pression de la revendication de menus halal qui n'hésite plus à user la voie juridique pour se faire entendre. Le succès en 2013 du recours administratif d'un détenu arguant du principe de laïcité pour contester le refus de l'établissement de lui servir un repas halal l'illustre ${ }^{4}$. La décision du tribunal administratif ${ }^{5}$ qui a fait suite à sa requête est révélatrice de la situation d'une administration prise aujourd'hui entre, d'un côté, les demandes de la population pénale en matière d'alimentation halal et, de l'autre, des pressions de plus en plus fortes des droits et de leurs gardiens, l'enjoignant à reconsidérer des arrangements institutionnels jugés insuffisamment respectueux du libre exercice du culte garanti par le principe de laïcité.

Après une brève description de la manière dont la DAP organise la prise en charge du particularisme alimentaire des musulmans, nous examinerons comment cette prise en charge, «minimaliste » (en comparaison ce qui se passe chez certains de nos voisins européens), semble

\footnotetext{
${ }^{4}$ Le Monde, 27 et 29/11/2013 ; Le Parisien, 27 et 29/11/2013 ; LBfmtv, 27/11/2013 ; Le Figaro, 28/11/2013 ; Libération, 29/11/2013 ; France Info, 29/11/2013 ; La Croix, 29/11/2013 ; Rue89, 29/11/2013, L'express, 29/11/2013; Le Nouvel Observation, 29/11/2013, etc.

5 Décision TA de Grenoble, 7 novembre 2013. M. AB c/ Directeur centre pénitentiaire de Saint-QuentinFallavier. Requête $\mathrm{n}^{\circ} 1302502$.
} 
aujourd'hui de plus en plus bousculée. Puis, dans un second temps, nous exposerons les diverses manières dont les individus s'identifiant à l'islam tentent, malgré les limites de l'offre institutionnelle, de s'acquitter des impératifs liés au halal. On s'attachera, ce faisant à ne pas détacher le sens de la revendication et/ou consommation de nourritures halal du contexte carcéral où elles prennent place. 